Mathematical Research Letters 1, 437-450 (1994)

\title{
STABILITY OF SPECTRAL TYPES FOR STURM-LIOUVILLE OPERATORS
}

\author{
R. Del Rio, B. Simon, And G. Stolz
}

\begin{abstract}
A вstract. For Sturm-Liouville operators on the half line, we show that the property of having singular, singular continuous, or pure point spectrum for a set of boundary conditions of positive measure depends only on the behavior of the potential at infinity. We also prove that existence of recurrent spectrum implies that of singular spectrum and that "almost sure" existence of $L_{2}$-solutions implies pure point spectrum for almost every boundary condition. The same results hold for Jacobi matrices on the discrete half line.
\end{abstract}

\section{Introduction}

For Sturm-Liouville operators generated by $-\frac{d^{2}}{d x^{2}}+q$ on the half line $[0, \infty)$, we study the dependence of spectral types on the boundary condition at 0 and on compactly supported perturbations of the potential. In Weidmann [17], it was conjectured that the existence of singular spectrum depends only on the behavior of the potential close to infinity. This, strictly speaking, is not true (see $[4,5]$ ). However, we now prove that existence of singular, singular continuous, or pure point spectrum for a set of boundary conditions of positive measure does not depend on the local behavior of the potential (Section 5).

Our proof of this result is prepared in Sections $2-3$ and relies mainly on:

(i) The identification of Aronszajn [1] and Donoghue [7] of the various parts of the spectrum under variation of boundary condition or rank one perturbation.

Received March 7, 1994.

The work of R. del Rio is partially supported by DGAPA-UNAM and CONACYT.

The work of R. del Rio and B. Simon is partially supported by the National Science Foundation under Grant No. DMS-9101715. The Government has certain rights in this material. 
(ii) A result on the average of the spectral measure with respect to boundary condition that goes back to Javrjan [9]; we use it in a form rediscovered by Kotani [12].

(iii) The invariance of the absolutely continuous spectrum under local perturbations.

(iv) Invariance of the set of energies with solutions $L^{2}$ at infinity under local perturbations.

There are two other interesting consequences of these basic results:

(i) A further subdivision of the subspace of absolute continuity of a self-adjoint operator was proposed by Avron-Simon [2], where the definitions of transient and recurrent subspaces were introduced. It was noticed in [2] that the recurrent spectrum is, in some sense, close to the singular continuous spectrum. In fact, as we shall see in Corollary 4.3, the presence of recurrent spectrum for some boundary condition implies the presence of singular spectrum for more than a null set of other boundary conditions.

(ii) The existence of $L_{2}$-solutions for (Lebesgue-) almost every value of the eigenvalue parameter implies pure point spectrum for almost every boundary condition (Corollary 3.2). This was noted already in [6, Theorem 5.1] and generalizes a result of Kirsch, Molchanov, and Pastur [11, Theorem 2], who applied this to potentials with an infinite number of (high or wide) barriers [10,11]. Further applications will be given in [16].

As noted in [8], there is a unified approach to rank one perturbations and variation of boundary condition if we consider perturbations

$$
A_{\alpha}=A+\alpha B
$$

with

$$
B \psi=(\varphi, \psi) \varphi
$$

where $\varphi \in \mathcal{H}_{-1}(A)$, the quadratic form dual in the scale of spaces associated to $A . \varphi$ is always assumed cyclic. (1.1) is then interpreted as a form sum. Variation of boundary condition is then obtained by defining $A$ to have Neumann boundary condition, $\varphi$ to be $\delta(x)$. If $\alpha=-\cot (\theta)$, then $A_{\alpha}$ has boundary condition

$$
u(0) \cos \theta+u^{\prime}(0) \sin \theta=0 .
$$

Details of this relationship, including the connection between the functions $F(E)$ and the Weyl $m$-functions, and other results we'll need are 
reviewed in Simon [14]. $\theta=0$, that is, $\alpha=\infty$ is discussed in GesztesySimon [8].

Note that this identification of variation of boundary condition with rank one perturbation of type (1.1) needs that the negative part of $q_{-}$

of the potential is infinitesimally form bounded with respect to $-\frac{d^{2}}{d x^{2}}$ on $L^{2}(0, \infty)$ with Neumann boundary condition at 0 ; compare [14]. Therefore, the application of our general results below to Sturm-Liouville operators needs that $q_{+} \in L_{\mathrm{loc}}^{1}(0, \infty)$ and, for example, $q_{-}$is bounded (more generally, $q_{-}$which are locally uniformly integrable are included). However, all our results on variation of the boundary condition or local perturbations for Sturm-Liouville operators are true under the weaker assumption that $-\frac{d^{2}}{d x^{2}}+q$ is limit point at infinity. In particular, this includes many operators which are not bounded from below. The proof uses the Weyl $m$-function and the Weyl spectral measure instead of the function $F_{\alpha}$ and measure $\mu_{\alpha}$ introduced below (for their relation, see [14]) and proceeds in almost complete analogy. Nevertheless, we use the rank one perturbation approach, which is more general in other respects. For example, it immediately shows that all our results for Sturm-Liouville operators have analogs for Jacobi matrices on the discrete half line and extends to the case where $q$ is singular at $x=0$, but not so singular that 0 stops being limit circle.

\section{Rank One Spectral Theory}

In the context of (1.1a), let $d \mu_{\alpha}$ be the spectral measure for $\varphi$ and $A_{\alpha}$, so

$$
F_{\alpha}(z) \equiv\left(\varphi,\left(A_{\alpha}-z\right)^{-1} \varphi\right)=\int \frac{d \mu_{\alpha}(x)}{(x-z)} .
$$

We set $F(z) \equiv F_{\alpha=0}(z)$.

It is also convenient to define

$$
d \rho_{\alpha}=\left(1+\alpha^{2}\right) d \mu_{\alpha}
$$

since $d \rho_{\infty}=\lim d \rho_{\alpha}$ then exists and [8]

$$
d \rho_{\infty}=\lim _{\epsilon \downarrow 0}\left[\pi^{-1} \operatorname{Im}(-F(x+i \epsilon))^{-1} d x\right] .
$$

$A_{\alpha}$ converges to a self-adjoint operator $A_{\infty}$ in norm resolvent sense [8]. In the half-line Sturm-Liouville case, $A_{\infty}$ is $-\frac{d^{2}}{d x^{2}}+q$ with Dirichlet boundary condition.

Moreover, since $\varphi$ is assumed cyclic, $A_{\alpha}$ is unitarily equivalent to multiplication by $x$ on $L^{2}\left(\mathbb{R}, d \rho_{\alpha}\right)$ if $\alpha \neq \infty$ and $A_{\infty}$ is equivalent to multiplication by $x$ on $L^{2}\left(\mathbb{R}, d \rho_{\infty}\right)[8]$. 
General principles imply $F(z)$ has boundary values $F(E+i 0)$ for a.e. $E$. Set $G(x)=\int \frac{d \mu_{0}(y)}{|x-y|^{2}}$. Note that $G(E)<\infty$ implies that $F(E+i 0)$ exists and is real [14].

The following is essentially in Aronszajn [1] and Donoghue [7]; see [14] for a short proof:

Theorem 2.1. $([1,7])$ For $\alpha \neq 0\left(\alpha=\infty\right.$ allowed with $\left.\infty^{-1}=0\right)$, define

$$
\begin{aligned}
S_{\alpha} & =\left\{x \in \mathbb{R} \mid F(x+i 0)=-\alpha^{-1} ; G(x)=\infty\right\} \\
P_{\alpha} & =\left\{x \in \mathbb{R} \mid F(x+i 0)=-\alpha^{-1} ; G(x)<\infty\right\} \\
L & =\{x \in \mathbb{R} \mid F(x+i 0) \text { exists and } \operatorname{Im} F(x+i 0) \neq 0\} .
\end{aligned}
$$

Then

(i) $\left\{S_{\alpha}\right\}_{\alpha \neq 0 ;|\alpha| \leq \infty},\left\{P_{\alpha}\right\}_{\alpha \neq 0 ;|\alpha| \leq \infty}$ and $L$ are mutually disjoint.

(ii) $P_{\alpha}$ is the set of eigenvalues of $A_{\alpha}$. In fact

$$
\begin{aligned}
\left(d \rho_{\alpha}\right)_{\mathrm{pp}}(x) & =\sum_{x_{n} \in P_{\alpha}} \frac{1+\alpha^{2}}{\alpha^{2} G\left(x_{n}\right)} \delta\left(x-x_{n}\right) & & \alpha<\infty \\
\left(d \rho_{\infty}\right)_{\mathrm{pp}}(x) & =\sum_{x_{n} \in P_{\infty}} \frac{1}{G\left(x_{n}\right)} \delta\left(x-x_{n}\right) & & \alpha=\infty .
\end{aligned}
$$

(iii) $\left(d \rho_{\alpha}\right)_{\mathrm{ac}}$ is supported on $L,\left(d \rho_{\alpha}\right)_{\mathrm{sc}}$ is supported on $S_{\alpha}$.

(iv) For $\alpha \neq \beta,\left(d \rho_{\alpha}\right)_{\operatorname{sing}}$ and $\left(d \rho_{\beta}\right)_{\text {sing }}$ are mutually singular.

In the above, we say $S$ "supports a measure $d \nu$ " if $\nu(\mathbb{R} \backslash S)=0$. And for any measure $d \nu$, we use $d \nu_{\mathrm{pp}}, d \nu_{\mathrm{ac}}, d \nu_{\mathrm{sc}}, d \nu_{\mathrm{sing}} \equiv d \nu_{\mathrm{pp}}+d \nu_{\mathrm{sc}}$ for the pure point, absolutely continuous, singular continuous, and singular parts of $d \nu$.

One can say more than that $L$ supports $\left(d \rho_{\alpha}\right)_{\text {ac }}$. Recall that any a.c. measure $d \nu(E)$ is of the form $f(E) d E$ and that $\{E \mid f(E) \neq 0\}$ which is a.e. defined is called the essential support of $d \nu$ (also called a minimal support). Since (see, e.g., [14]):

$$
\begin{aligned}
d \rho_{\alpha, \mathrm{ac}} & =\frac{1+\alpha^{2}}{\pi} \operatorname{Im} F_{\alpha}(E+i 0) d E \quad \alpha<\infty \\
\operatorname{Im} F_{\alpha}(z) & =\operatorname{Im} F(z) / \mid\left(1+\left.\alpha F(z)\right|^{2}\right. \\
d \rho_{\infty, \mathrm{ac}}(E) & =-\frac{1}{\pi} \operatorname{Im} F_{0}(E+i 0)^{-1} d E,
\end{aligned}
$$

we see 
Theorem 2.2. The set $L$ of Theorem 2.1 is the essential support of each $\left(d \rho_{\alpha}\right)_{\mathrm{ac}}$.

It is useful to have $\alpha$ independent sets:

Corollary 2.3. Let

$$
\begin{aligned}
P & =\{x \mid G(x)<\infty\} \cup\{x \mid x \text { is an eigenvalue of } A\} \\
L & =\{x \mid F(x+i 0) \text { exists and } \operatorname{Im} F(x+i 0) \neq 0\} \\
S & =\mathbb{R} \backslash(P \cup L) .
\end{aligned}
$$

Then for $\alpha$ (including $\alpha=0$ and $\infty)$ :

$$
\left(d \rho_{\alpha}\right)_{\mathrm{ac}}=\chi_{L} d \rho_{\alpha} ; \quad\left(d \rho_{\alpha}\right)_{\mathrm{pp}}=\chi_{P} d \rho_{\alpha} ; \quad\left(d \rho_{\alpha}\right)_{\mathrm{sc}}=\chi_{S} d \rho_{\alpha}
$$

Proof. For $\alpha \neq 0$, this follows immediately from $P_{\alpha} \subset P, S_{\alpha} \subset S$ and Theorem 2.1. For $\alpha=0, P$ contains the eigenvalues by construction, $L$ supports $d \rho_{\alpha=0, \text { ac }}$ by $(2.1)$, and $S$ supports $d \rho_{\alpha=0, \text { sc }}$. Since $S$ contains $\left\{E\left|\lim _{\epsilon \downarrow 0}\right| F(E+i \epsilon) \mid=\infty\right\} \backslash\{E \mid E$ is an eigenvalue of $A\}$.

Proposition 2.4. The sets, $P, S, L$ are $\alpha$ independent, that is, one obtains the same sets starting from any $A_{\alpha},|\alpha|<\infty$.

Proof. (2.1b) and the related $F_{\alpha}(E)=F(E) / 1+\alpha F(E)$ show that $L$ is independent of $\alpha$. If $G(E)<\infty$, then $F(E+i 0)$ has a real value, so $E$ is actually an eigenvalue of $\left\{A_{\alpha}\right\}_{\alpha \neq 0}$ with $\alpha=\infty$ allowed (if $F(E+i 0)=0$ ). Using also Theorem 2.1(ii), we get that $P=\cup\left\{E \mid E\right.$ is an eigenvalue of $A_{\alpha} ; \alpha \in$ $\mathbb{R} \cup\{\infty\}\}$. Since $\left\{A_{\beta+\alpha}\right\}=\left\{A_{\beta}\right\}$ for any fixed $\alpha, P$ is $\alpha$ independent. Thus, $S=\mathbb{R} \backslash(L \cup P)$ is also $\alpha$ independent.

The following integral relation is a result of Javrjan [9]; see also Kotani [12] and Simon-Wolff [15].

Theorem 2.5. For any Borel set $M$, we have that

$$
\begin{aligned}
\int \mu_{\alpha}(M) d \alpha & =|M| \\
\int \rho_{\cot (\theta)}(M) d \theta & =|M|
\end{aligned}
$$

where $|M|$ is the Lebesgue measure of $M$. 

that

It follows from the fact that the sets in Corollary 2.3 are $\alpha$ independent

Theorem 2.6. For any Borel set $M$, we have that

$$
\begin{aligned}
\int \mu_{\alpha, \mathrm{pp}}(M) d \alpha & =|M \cap P| \\
\int \mu_{\alpha, \mathrm{sc}}(M) d \alpha & =|M \cap S| \\
\int \mu_{\alpha, \mathrm{sing}}(M) d \alpha & =|M \cap(S \cup P)| \\
\int \mu_{\alpha, \mathrm{ac}}(M) d \alpha & =|M \cap L|
\end{aligned}
$$

Thus, we immediately have:

Corollary 2.7. For any Borel set $M, \mu_{\alpha, \mathrm{pp}}(M) \neq 0$ for some set of $\alpha$ 's of positive measure if and only if $|M \cap P| \neq 0$ and similarly for $\mu_{\alpha, \mathrm{sc}}(M)$ and $|M \cap S|$, and for $\mu_{\alpha, \text { sing }}(M)$ and $M \cap(S \cup P)$. In particular, $\mu_{\alpha, \mathrm{sc}} \not \equiv 0$ for a set of $\alpha$ 's of positive measure if and only if $|S| \neq 0$.

If one wants to state this theorem in terms of spectrum, one has to face the fact that the spectrum is a poor invariant for measures, so one is restricted to open sets.

Corollary 2.8. For any open set $I, I \cap \sigma_{\alpha, \mathrm{sc}} \neq \emptyset$ for a set of $\alpha$ 's of positive measure if and only if $|I \cap S| \neq 0$ and similarly for $\sigma_{\alpha, \mathrm{pp}}$ and $\sigma_{\alpha, \mathrm{sing}}$.

Proof. For an open set $I$, and arbitrary measure $d \nu, I \cap \operatorname{supp}(d \nu) \neq \emptyset$ if and only if $\nu(I) \neq 0$.

Example. Suppose $A$ has only point spectrum in $(-\infty, 0)$ and an infinity of eigenvalues $e_{1}<e_{2}<\cdots<e_{n}<\cdots$ with $\lim e_{n}=0$. Then by standard intertwining, each $A_{\alpha}$ has an infinity of eigenvalues, one in each $\left(e_{n}, e_{n+1}\right)$ and so $0 \in \sigma_{\mathrm{pp}}\left(A_{\alpha}\right)$ for all $\alpha$, so Corollary 2.8 fails for $I=\{0\}$. Of course, Corollary 2.7 is still valid.

\section{Point Spectrum in the Sturm-Liouville Case}

We have seen that a special role is played by the set

$$
P=\{E \mid G(E)<\infty\} \cup\{\text { eigenvalues of } A\} .
$$

In the Sturm-Liouville case, we want to identify $P$ : 
Theorem 3.1. Let $A=-\frac{d^{2}}{d x^{2}}+q(x)$ be limit point at infinity and suppose that $A$ is defined with Neumann boundary conditions. Then

$$
P=\left\{E \in \mathbb{R} \mid-u^{\prime \prime}+q(x) u=E u \text { has a solution } L^{2} \text { at } x=+\infty\right\} .
$$

Proof. As already noted in the proof of Proposition 2.4, $P=\cup\{E \mid E$ is an eigenvalue of some $\left.A_{\alpha} ; \alpha \in \mathbb{R} \cup\{\infty\}\right\}$. But since every solution $L^{2}$ at $\infty$ obeys some boundary value at 0 , this is precisely the set of $E$ 's with a solution $L^{2}$ at $\infty$.

Corollary 3.2. Let $I$ be an open set in $\mathbb{R}$. If for a.e. $E \in I,-u^{\prime \prime}+q(x) u=$ Eu has a solution which is $L^{2}$ at $\infty$, then for a.e. boundary condition, $-\frac{d^{2}}{d x}+q$ with that boundary condition has only point spectrum in $I$.

\section{Transient and Recurrent Spectrum}

Definition ([2]). A vector $\varphi \in \mathcal{H}$ is called a transient vector for $H$ if and only if for all $N>0$,

$$
\lim _{|t| \rightarrow \infty}|t|^{N}\left(\varphi, e^{-i t H} \varphi\right)=0 .
$$

The transient subspace $\mathcal{H}_{\mathrm{tac}}$ is the closure of the set of transient vectors. We have $\mathcal{H}_{\text {tac }} \subset \mathcal{H}_{\mathrm{ac}}$. The recurrent space $\mathcal{H}_{\text {rac }}$ is the orthogonal complement of $\mathcal{H}_{\text {tac }}$ in $\mathcal{H}_{\mathrm{ac}}$, that is, $\mathcal{H}_{\text {rac }}=\mathcal{H}_{\mathrm{ac}} \cap \mathcal{H}_{\text {tac }}^{\perp}$.

Lemma 4.1. Let I be an open subset of $\mathbb{R}$. Suppose that $A$ is multiplication by $x$ on $L^{2}(\mathbb{R}, d \mu)$ and

$$
d \mu_{\mathrm{ac}}(x)=f(x) d x
$$

with $f>0$ a.e. on $I$. Then $E_{I} \mathcal{H}_{\mathrm{rac}}=0$ where $E_{I}$ is the spectral measure for $A$.

Proof. Let $U: L^{2}(I, d x) \rightarrow L^{2}(I, f(x) d x)$ by

$$
(U g)(x)=f^{-1 / 2}(x) g x .
$$

$U$ sets up a unitary equivalence between multiplication by $x$ on $L^{2}(I, d x)$ and $A \uparrow E_{I} \mathcal{H}_{\mathrm{ac}}$. That all vectors are transient follows from Proposition 3.3 and Example 3.8 in [2].

Theorem 4.2. In the context of rank one perturbations, suppose $I \subset \mathbb{R}$ is open and $A_{\alpha}$ has only absolutely continuous spectrum in I for a.e. $\alpha$. Then for any $\alpha$, the spectrum is purely transient in $I$.

Proof. By hypothesis and Theorem 2.6, $|I \cap P|=|I \cap S|=0$ so $|I \triangle L|=0$; that is, Lemma 4.1 applies for any $A_{\alpha}$ and so $E_{I} \mathcal{H}_{\text {rac }}=0$. 
Corollary 4.3. Suppose $A$ is self-adjoint and $I \subset \mathbb{R}$ is open. Suppose $\varphi$ is cyclic for $A$ and that $E_{I} \mathcal{H}_{\mathrm{rac}} \neq 0$. Then for a set of $\alpha$ 's of positive measure, $A_{\alpha}$ has singular spectrum in $I$.

Note that this result does not say if the singular spectrum is point or singular continuous. As we'll see in Section 6, either can occur.

\section{Local Perturbations}

In this section, we prove our main new result that the occurrence of any specific spectral type for a set of positive measures of boundary conditions for a Sturm-Liouville operator is invariant under local perturbations of potential.

Let $q$ be locally in $L^{1}$ on $[0, \infty)$ be such that $-\frac{d^{2}}{d x^{2}}+q$ is limit point at $\infty$ and let $H_{\theta}(q)$ be the operator $-\frac{d^{2}}{d x^{2}}+q$ with boundary condition (1.2) at 0 (see [3] for the precise definition). For $\theta \neq 0$, let $S_{\theta}(q), P_{\theta}(q), L_{\theta}(q)$ be the set defined in Corollary 2.3 for $\varphi=\delta_{0}$ and $A=H_{\theta}(q)$. We already know (Proposition 2.4) that these sets are $\theta$ independent. Here we note that

Theorem 5.1. Let $v$ be in $L^{1}$ with compact support. Then

$$
\begin{gathered}
\left|S_{\theta}(q+v) \triangle S_{\beta}(q)\right|=0 \\
P_{\theta}(q+v)=P_{\beta}(q) \\
\left|L_{\theta}(q+v) \triangle L_{\beta}(q)\right|=0
\end{gathered}
$$

for any $\theta, \beta$.

Proof. As already noted, the sets are $\theta$ independent and since $S=\mathbb{R} \backslash(P \cup$ $L)$, we need only prove the results $(5.2),(5.3)$. (5.2) follows immediately from Theorem 3.1 and the fact that solutions of $-u^{\prime \prime}+q u=e u$ and $-w^{\prime \prime}+$ $(q+v) w=e w$ agree near infinity.

To prove (5.3), let $\operatorname{supp} v \subset[0, c]$. Let $A=-\frac{d^{2}}{d x^{2}}+q$ with Neumann boundary condition at 0 ; let $B=A+v$; let $\tilde{A}$ be $A$ with an additional Dirichlet boundary at $c$, and similarly for $\tilde{B}$. Then $(\tilde{A}+i)^{-1}-(A+i)^{-1}$ and $(\tilde{B}+i)^{-1}-(B+i)^{-1}$ are rank one. Moreover, $(\tilde{A}+i)^{-1}=\left(\tilde{A}_{\mathrm{in}}+i\right)^{-1} \oplus$ $\left(\tilde{A}_{\text {out }}+i\right)^{-1}$ on $L^{2}(0, c) \oplus L^{2}(c, \infty)$ with $\left(\tilde{A}_{\text {in }}+i\right)^{-1}$ compact, and similarly for $B$. Moreover, $\tilde{A}_{\text {out }}=\tilde{B}_{\text {out }}$. Thus, $A$ and $B$ have unitarily equivalent a.c. subspaces (by using the Kuroda-Birman theorem (see, e.g., [13])). Since $L$ is an essential support of the a.c. part of the spectral measure, we see that $L_{\theta}(q)$ and $L_{\theta}(q+v)$ agree up to sets of measure zero. 
Corollary 5.2. Let $I \subset \mathbb{R}$ be open. If $L_{\theta}(q)$ has singular continuous spectrum in I for a set of positive measure of $\theta$ 's, the same is true for $q+v$.

Proof. Follows from Corollary 2.8 and Theorem 5.1.

Similar results hold for point spectrum and singular spectrum.

\section{Examples}

Here are five examples closely related to Examples 1-3 in Simon-Wolff [15] and Appendix 2 of [2]. They illustrate the kinds of spectrum that can appear under rank one perturbations $A+\alpha(\varphi, \cdot) \varphi$ when $A$ either has recurrent a.c. spectrum (Examples 1, 4, 5) or transient a.c. spectrum (Examples $2,3)$. In Example 1 the singular spectrum appearing under rank one perturbations is just discrete eigenvalues, but lying outside the a.c. spectrum. Examples 4 and 5 are somewhat more interesting in providing situations where either s.c. spectrum or eigenvalues appear embedded in the recurrent a.c. spectrum. Examples 2 and 3 show that there is no converse to Corollary 4.3; namely, that the existence of singular spectrum for a set of positive measure $\alpha$ 's does not imply that $A$ has any recurrent spectrum, even if the a.c. spectrum has full support.

In all cases we can totally describe the example by giving the spectral measure $d \mu_{A}^{\varphi}$, which we'll call $d \mu$. In each case we'll take $d \mu(x)=\chi_{B}(x) d x$ where $B$ is a Lebesgue measurable set.

Example 1. Take $B$ to be a positive measure Cantor-type set. For example, start with $[0,1]$, remove the middle $\left(\frac{1}{n_{j}}\right)$, the fraction at step $j$ with $n_{j}=j^{2}$. As usual, $B$ is a closed nowhere dense set. Let $F(z)=$ $\int_{B}(x-z)^{-1} d x$. Then it is easy to see that $\varlimsup_{\epsilon \downarrow 0} \operatorname{Im} F(x+i 0)>0$ on $B$; indeed, we believe $\lim _{\epsilon \downarrow 0} \operatorname{Im} F(x+i \epsilon)$ is $\frac{1}{2}$ if $x$ is a boundary point of a connected component of $[0,1] \backslash B$ and is 1 otherwise. Because $\varlimsup \lim \operatorname{Im} F>0$ for all $x$ in $B, d \mu_{\alpha, \operatorname{sing}}(B)=0$ for all $\alpha$. $A_{\alpha}$ for $\alpha \neq 0$ has a.c. spectrum $B$, and a single eigenvalue in each component of $[0,1] \backslash B$. In this case the singular spectrum guaranteed by Corollary 4.3 is just discrete eigenvalues.

Example 2. Let $\left\{q_{n}\right\}_{n=1}^{\infty}$ be a counting of the rationals. Let $a<\frac{1}{2}$ and let $B=[0,1] \cap\left[\bigcup_{n=1}^{\infty}\left(q_{n}-\frac{a^{n}}{2}, q_{n}+\frac{a^{n}}{2}\right)\right]$. Then $|[0,1] \backslash B|>1-\left(a+a^{2}+a^{3} \ldots\right)=$ $(1-2 a) /(1-a)$ is a closed nowhere dense set of positive Lebesgue measure. It is easy to see that $G(x)<\infty$ for a.e. $x$ in $[0,1] \backslash B$ by the argument in Example 3 in [15]. Thus, since $|[0,1] \backslash B|>0$, we know that for a set of $\alpha$ 's of positive measure, $A_{\alpha}$ has eigenvalues in $[0,1]$. Of course, $\sigma_{\mathrm{ac}}=\bar{B}=[0,1]$. 
Example 3. Let $B=\left[\bigcup_{n=1}^{\infty} \bigcup_{j=1}^{2^{n}}\left[\frac{1}{2^{n}}\left(1-\frac{1}{4} n^{-2}\right), \frac{1}{2^{n}}\left(1+\frac{1}{4} n^{-2}\right)\right]\right] \cap[0,1]$. Then $|[0,1] \backslash B| \geq 1-\frac{1}{2} \sum_{n=1}^{\infty} n^{-2}>0$ so $[0,1] \backslash B$ is a closed nowhere dense set of positive Lebesgue measure. As in Example 2 in [15], $G(x)=\infty$ on all of $[0,1]$ so no $A_{\alpha}$ has point spectrum in $[0,1]$. By Theorem 2.6, for a set of $\alpha$ 's of positive measure, $A_{\alpha}$ has some singular continuous spectrum embedded in $\sigma_{\mathrm{ac}}\left(A_{\alpha}\right)=[0,1]$.

For Examples 4 and 5 , let $n_{j}=2 \ell_{j}+1$ be a sequence of odd integers with

$$
\sum_{j=1}^{\infty} n_{j}^{-1}<\infty
$$

As in Appendix 2 of [2], we can define functions $a_{j}(x)$ for $x \in\left[-\frac{1}{2},-\frac{1}{2}\right]$ with $a_{j} \in\left\{-\ell_{j},-\ell_{j}+1, \ldots, \ell_{j}-1, \ell_{j}\right\}$ by using a variable base expansion:

$$
x=\sum_{j=1}^{\infty} \frac{a_{j}(x)}{n_{1} \ldots n_{j}} .
$$

Lebesgue measure corresponds to taking the $a_{j}$ 's independent with uniform distribution among the $n_{j}$ values. By (6.1) and the Borel-Cantelli lemma, $\mid\left\{x \mid a_{j}(x)=0\right.$ for infinitely many $j$ 's $\} \mid=0$. As in [2], define

$$
\begin{aligned}
& B=\left\{x \mid a_{j}(x)=0 \text { for an odd number of } j \text { 's }\right\} \\
& C=\left\{x \mid a_{j}(x)=0 \text { for an even number or for infinitely many } j \text { 's }\right\} .
\end{aligned}
$$

We'll also define

$$
\begin{aligned}
D & =\left\{x|| a_{j}(x) \mid \geq 2 \text { all } j\right\} \\
B_{j} & =\left\{x \mid a_{j}(x)=0\right\} \\
S_{j}(y) & =\left\{x|| x-y \mid \leq \frac{1}{n_{1} \ldots n_{j}}\right\} .
\end{aligned}
$$

We note first that $|D|>0$ if all $n_{j} \geq 5$ since

$$
|D|=\prod_{j=1}^{\infty}\left(1-\frac{3}{n_{j}}\right)>0
$$

by $(6.1)$. 
Next (following the argument in [2]):

$$
\begin{aligned}
& \left|S_{j}(y) \cap B\right| \geq \gamma\left|S_{j}(y)\right|\left(n_{j+1}\right)^{-1} \\
& \left|S_{j}(y) \cap C\right| \geq \gamma\left|S_{j}(y)\right|\left(n_{j+1}\right)^{-1}
\end{aligned}
$$

so long as $y \neq \pm \frac{1}{2}$ and $j$ is so large that $S_{j}(y) \subset(-1,1)$. In $(6.4) \gamma$ is the fixed constant

$$
\gamma=\frac{1}{2} \prod_{\ell=1}^{\infty}\left(1-\frac{1}{n_{\ell}}\right)>0
$$

To prove (6.4), note first that

$$
a_{\ell}(x)=a_{\ell}(y) ; \quad \ell=1, \ldots, j \Longrightarrow x \in S_{j}(y)
$$

Suppose $\#\left\{\ell \leq j \mid a_{\ell}(y)=0\right\}$ is odd. Then

$$
S_{j}(y) \cap B \supset\left\{x \mid a_{\ell}(x)=a_{\ell}(y), \ell=1, \ldots, j ; \quad a_{\ell}(x) \neq 0, \ell>j\right\}
$$

which has measure

$$
\left(n_{1} \ldots n_{j}\right)^{-1} \prod_{\ell=j+1}^{\infty}\left(1-\frac{1}{n_{\ell}}\right) \geq \frac{1}{2}\left|S_{j}(y)\right| \prod_{\ell=1}^{\infty}\left(1-\frac{1}{n_{\ell}}\right)
$$

while

$$
\begin{aligned}
& S_{j}(y) \cap C \supset \\
& \quad\left\{x \mid a_{\ell}(x)=a_{\ell}(y), \ell=1, \ldots, j ; \quad a_{j+1}(x)=0, a_{\ell}(x) \neq 0, \ell>j+1\right\}
\end{aligned}
$$

which has measure

$$
\left(n_{1} \ldots n_{j}\right)^{-1} n_{j+1}^{-1} \prod_{\ell=j+1}^{\infty}\left(1-\frac{1}{n_{\ell}}\right) \geq \gamma\left|S_{j}(y)\right| / n_{j+1}
$$

A similar argument applies if $\#\left\{\ell \leq j \mid a_{\ell}(y)=0\right\}$ is even.

As a final preliminary we need that

$$
\inf \left\{|x-y| \mid x \in D, y \in B_{j}\right\}=\left(n_{1} \ldots n_{j}\right)^{-1}
$$


Example 4. Pick $n_{j}$ so that (6.1) holds and

$$
\lim _{j \rightarrow \infty} \frac{n_{1} \ldots n_{j}}{n_{j+1}}=\infty
$$

for example, $\ell_{j}=2^{j}$. Let $d \mu=\chi_{B} d x$. Then we claim $G(y)=\infty$ for all $y$. For

$$
\begin{aligned}
G(y) & \geq\left|S_{j}(y) \cap B\right|\left(n_{1} \ldots n_{j}\right)^{2} \\
& \geq 2 \gamma \frac{n_{1} \ldots n_{j}}{n_{j+1}}
\end{aligned}
$$

by $(6.4 \mathrm{a})$. Moreover by $(6.4 \mathrm{a}, \mathrm{b})$ the essential closure of $B$ is $\left[-\frac{1}{2}, \frac{1}{2}\right]$ and the essential closure of $\left[-\frac{1}{2}, \frac{1}{2}\right] \backslash B$ is also $\left[-\frac{1}{2}, \frac{1}{2}\right]$. Thus, the operator $A$ has recurrent spectrum $\left[-\frac{1}{2}, \frac{1}{2}\right]$ and no $A_{\alpha}$ has point spectrum. Since $\left|\left[-\frac{1}{2}, \frac{1}{2}\right] \backslash B\right|=|C|>0$ for a positive measure set of $\alpha$ 's, $A_{\alpha}$ has singular continuous spectrum embedded in $\left[-\frac{1}{2}, \frac{1}{2}\right]=\sigma_{\mathrm{ac}}\left(A_{\alpha}\right)$.

Example 5. Pick $n_{j}$ so that

$$
\sum_{j=1}^{\infty}\left(\frac{n_{1} \ldots n_{j}}{n_{j+1}}\right)^{2}<\infty
$$

and that

$$
\sum_{j=k+1}^{\infty} \frac{1}{n_{j}} \leq \frac{1}{n_{k}},
$$

for example, $n_{j}=2^{j !}$. Define $\tilde{B}$ analogously to $B$ but with

$$
\tilde{B}=\left\{x \mid \text { the number of } j \text { with } a_{j}(x)=0 \text { lies in }\{3,5,7, \ldots\}\right\}
$$

and let $d \mu=\chi_{\tilde{B}} d x$. As above, $A$ has recurrent spectrum with essential support $\tilde{B}$ but closed support $\left[-\frac{1}{2}, \frac{1}{2}\right]$. We claim that $G(x)<\infty$ on $D$. Since $|D|>0, A_{\alpha}$ has point spectrum embedded in $\left[-\frac{1}{2}, \frac{1}{2}\right]=\sigma_{\mathrm{ac}}$ for a set of $\alpha$ 's of positive measure. Note that this does not exclude the occurrence of singular spectrum in addition.

To see that $G(x)<\infty$, define

$$
B_{j, k, \ell}=\left\{x \mid a_{j}(x)=a_{k}(x)=a_{\ell}(x)=0\right\} .
$$


Thus by (6.8),

$$
\sum_{\substack{k, \ell>j \\ k \neq \ell}}\left|B_{j, k, \ell}\right| \leq\left(\sum_{m=j+1}^{\infty} \frac{1}{n_{m}}\right)^{2} \frac{1}{n_{j}} \leq \frac{4}{n_{j}\left(n_{j+1}\right)^{2}}
$$

since (6.8) implies that

$$
\sum_{j=k+1}^{\infty} \frac{1}{n_{j}} \leq \frac{2}{n_{k+1}}
$$

On the other hand, by (6.5),

$$
\inf \left\{|x-y| \mid x \in D, y \in B_{j, k, \ell}\right\} \geq\left(n_{1} \ldots n_{j}\right)^{-1} \quad k, \ell>j .
$$

Since $\tilde{B} \subset \underset{\substack{j, k, \ell \\ \text { all unequal }}}{\cup} B_{j, k, \ell}$, we have by (6.9-6.10) that if $x \in D$

$$
G(x) \leq 4 \sum_{j=1}^{\infty} \frac{\left(n_{1} \ldots n_{j}\right)^{2}}{n_{j}\left(n_{j+1}\right)^{2}}<\infty
$$

by $(6.7)$.

\section{Acknowledgments}

R. del Rio would like to thank J. Weidmann for having initiated him in the study of stability of spectral types. R. del Rio and G. Stolz would also like to thank C. Peck and M. Aschbacher for the hospitality at Caltech.

\section{References}

[1] N. Aronszajn, On a problem of Weyl in the theory of singular Sturm-Liouville equations, Amer. J. Math. 79 (1957), 597-616.

[2] J. Avron and B. Simon, Transient and recurrent spectrum, J. Funct. Anal. 43 (1981), 1-31.

[3] B.A. Coddington and N. Levinson, Theory of Ordinary Differential Equations, McGraw-Hill, 1955.

[4] R. del Rio, Instability of the absolutely continuous spectrum of ordinary differential operators under local perturbations, J. Math. Anal. Appl. 142 (1989), 591-604.

[5] _ Ein Gegenbeispiel zur Stabilität des absolut stetigen Spektrums gewöhnlicher Differentialoperatoren, Math. Z 197 (1988), 61-68.

[6] R. del Rio, N. Makarov, and B. Simon, Operators with singular continuous spectrum, II. Rank one operators, Commun. Math. Phys. (to appear).

[7] W. Donoghue, On the perturbation of the spectra, Commun. Pure Appl. Math. 18 (1965), 559-579. 
[8] F. Gesztesy and B. Simon, Rank one perturbations at infinite coupling, J. Funct. Anal. (to appear).

[9] V.A. Javrjan, A certain inverse problem for Sturm-Liouville operators, Izv. Akad. Nauk Armjan. SSR Ser. Mat. 6 (1971), 246-251.

[10] W. Kirsch, S. Molchanov, and L. Pastur, One-dimensional Schrödinger operators with unbounded potential: The pure point spectrum, Funct. Anal. Appl. 24 (1990), 176-186.

[11] _ One-dimensional Schrödinger operators with high potential barriers, Operator Theory: Advances and Applications, vol. 57, Birkhäuser, 1992, pp. 163-170.

[12] S. Kotani, Lyapunov exponents and spectra for one-dimensional random Schrödinger operators, Contemp. Math. 50 (1986), 277-286.

[13] M. Reed and B. Simon, Methods of Modern Mathematical Analysis, III. Scattering Theory, Academic Press, 1979.

[14] B. Simon, Spectral analysis of rank one perturbations and applications, Proc. 1993 Vancouver Summer School in Mathematical Physics (to appear).

[15] B. Simon and T. Wolff, Singular continuous spectrum under rank one perturbations and localization for random Hamiltonians, Commun. Pure Appl. Math. 39 (1986), 75-90.

[16] G. Stolz, In preparation.

[17] J. Weidmann, Absolut stetiges Spektrum bei Sturm-Liouville Operatoren und Dirac Systemen, Math. Z. 180 (1982), 423-426.

IIm A S-UnAm, A pdo. Postal 20-726, Admon. No. 20, 01000 Mexico D.F., M EXICO.

E-mail address: delrio@redvax1.dgsca.unam.mx

Division of Physics, Mathematics, and Astronomy, California institute of TeChology, 253-37, Pasadena, CA 91125.

E-mail address: bsimon@caltech.edu

Fachbereich Math., Johann W olfgang Goethe-Universität, D-60054

Frankfurt am Main, Germany.

E-mail address: stolz@mathematik.uni-frankfurt.d400.de 